\title{
Baryon resonances in a finite volume
}

\author{
Ulf-G. Meißner ${ }^{1,2, \star}$ and Akaki Rusetsky ${ }^{1, \star \star}$ \\ ${ }^{1}$ Helmholtz-Institut für Strahlen- und Kernphysik (Theorie) and \\ Bethe Center for Theoretical Physics, Universität Bonn, D-53115 Bonn, Germany \\ ${ }^{2}$ Institute for Advanced Simulation (IAS-4), Institut für Kernphysik (IKP-3) and \\ Jülich Center for Hadron Physics, Forschungszentrum Jülich, D-52425 Jülich, Germany
}

\begin{abstract}
We review the main results obtained in the project A.6, which dealt with hadron-hadron scattering and resonances in a finite volume. These results provide tools for the extraction of the resonance characteristics from lattice QCD simulations. In particular, in this review we focus on the evaluation of the matrix elements of the weak and electromagnetic currents between the states containing at least one resonance, as well as on the description of the three-particle states in a finite volume. We also discuss insights obtained using unitarized chiral perturbation theory in a finite volume.
\end{abstract}

\section{Introduction}

The project A.6 has emerged from the project C.3 "Spin structure of the nucleon (theory)," and covers the last two funding periods of the SFB/TR 16 (2008-2016). The necessity to start this project was justified by the intensified efforts to study the properties of resonances by using lattice QCD. It should be pointed out that Monte Carlo simulations in lattice QCD constitute only a part of these efforts. In addition, one needs a framework, where the "raw" results of the simulations should be interpreted in terms of the observable hadronic characteristics, which can be directly compared to experiments. Without possessing tools for such an interpretation, the simulations alone might turn out to be useless.

In order to illustrate this statement, note first that a physical stable particle corresponds to an eigenstate of the finite-volume lattice Hamiltonian (let us remind the reader that the lattice simulations are always performed in a finite volume). Further, finite-volume artifacts in the measured values of the energies are usually small, being exponentially suppressed at large volumes. Thus, one may directly extract the masses of the stable particles from the results of simulations. On the other hand, resonances correspond to the poles of the $S$-matrix at complex energies on unphysical Riemann sheets, and not to the isolated eigenvalues of the Hamiltonian. Consequently, measuring the individual energy levels on the lattice does not immediately provide information about the location of a resonance pole. In order to extract the latter, one should first use a formula which relates the energy, measured in a finite volume, to the value of the infinite-volume phase at the same energy (this relation goes under the name of the Lüscher

\footnotetext{
^e-mail: meissner@hiskp.uni-bonn.de

${ }^{\star}$ e-mail: rusetsky@hiskp.uni-bonn.de
}

formula [1]). In the next step, using the extracted phase shifts at the different energies, one performs an analytic continuation into the complex plane and determines the position of the pole.

The above example clearly demonstrates the need of a framework, which should be used for the analysis of the lattice data. In case of more complicated systems (coupled-channel scattering, calculation of the matrix elements with resonances, many-particle systems), more elaborate tools are needed. In our study of these systems, we have extensively used non-relativistic effective field theories (EFT) in a finite volume, which enable one to systematically relate the measured quantities in a finite and in the infinite volume. The Lüscher formula, mentioned above, directly follows from this approach in the simple case of the elastic scattering. We briefly review the EFT approach in Sect. 2.

During 2008-2016, the investigations carried out within the project A.6, contributed significantly to the major developments in the field. In particular, we studied the extraction of the mass and width of the $\Delta$-resonance from the lattice data, performing the finite-volume calculations in Chiral Perturbation Theory (ChPT) at order $\epsilon^{4}$ within the Small Scale Expansion (SSE) [2]. The grouptheoretical aspects of the Lüscher approach, including the full analysis in the moving frames for the particles with both the integer and half-integer spin were addressed in Refs. [3, 4]. In Refs. [5, 6], using the non-relativistic EFT in a finite volume, we have generalized the Lüscher approach to the case of the coupled channels and discussed the extraction of the $\bar{K} N$ scattering lengths from the data, as well as the application in the scalar meson sector. A further development of these ideas are contained in Refs. [710], where the finite-volume generalization of the Unitary ChPT (UChPT) has been introduced. The parameters of 
UChPT are fitted to the measured energy levels in a finite volume. At the next stage, UChPT in the infinite volume with the same set of the parameters is used to reproduce the $S$-matrix elements and determine the position of its poles in the complex plane. Fitting the synthetic data, it was demonstrated that the method is very robust and works even if the data have sizable statistical errors. The main insights from this framework are summarized in Sec. 5.

We would like to stress that using an effective field theory approach in a finite volume has led to the two completely new developments in the field. The first one is the extraction of the resonance matrix elements [11-14] - by this, we mean the matrix elements of currents sandwiched by the states containing at least one resonance (we shall give a precise definition of this quantity in Sect. 3). Examples are given by the form factors of the $\Delta$ and $\rho$, the $\Delta N \gamma^{*}$ transition form factor, as well as the semileptonic form factors in the $B \rightarrow K^{*} \ell^{+} \ell^{-}$decays. The second development deals with the study of the three-particle systems in a finite volume [15-20]. These investigations, which are of ultimate importance for the analysis of the inelastic resonances (e.g., the Roper resonance) as well as the data obtained in nuclear lattice simulations, will be addressed in Sect. 6 in more detail.

Finally, it should be pointed out that there have been very interesting developments in using the non-relativistic EFT in the infinite volume. In particular, it has proven to be very useful in study of the three-particle decays [2123], $K_{\ell 4}$ decays [24], as well as in study of hadronic atoms $[25,26]$. Further developments include the study of the various aspects of the chiral expansion in the infinite volume [27-31], as well as the formulation of the alternative method for the extraction of a resonance mass and width on the lattice [32].

Disclaimer: Note that the present work is the report on the activities carried out within the project A.6 during the period 2008-2016. It is impossible to give all references relevant for the subjects covered by the research in eight years. Below, we shall mainly cite the work done within A.6, including a few more references whenever necessary. No claim is made on having a full list of references. We also point towards the connection to other projects.

\section{Non-relativistic effective theories}

In order to extract the hadron characteristics in the scattering sector from lattice data, the size of the lattice, $L$, should obey certain constraints. Namely, $L$ must be much larger than $R$, a typical scale of the hadronic interactions. Usually, $R$ is defined by the mass of the lightest particle in the system, which in the case of QCD is the pion mass. Consequently, one should work in the limit $M_{\pi} L \gg 1$. In the case of boxes of such size, the wave function of a system in the vicinity of the walls of the box has already its asymptotic form which, for elastic scattering, is determined solely by the scattering phase shift. Consequently, the discrete energy spectrum in a finite volume, which is obtained by imposing (periodic) boundary conditions on the wave function at the walls, is determined solely by the phase shift at the same energy. This statement conveys the main message contained in the Lüscher equation [1] which, in the S-wave and in the absence of the partialwave mixing, takes the simple form

$$
\begin{aligned}
\tan \delta_{0}(p) & =-\tan \phi(q), \\
\tan \phi(q) & =-\frac{\pi^{3 / 2} q}{Z_{00}\left(1 ; q^{2}\right)}, \quad q=\frac{p L}{2 \pi} .
\end{aligned}
$$

Here, $\delta_{0}(p)$ is the S-wave elastic scattering phase and $p$ denotes the magnitude of the relative three-momentum in the center-of-mass $(\mathrm{CM})$ frame. Further, $Z_{00}\left(1, q^{2}\right)$ stands for the Lüscher zeta-function

$$
Z_{00}\left(1 ; q^{2}\right)=\frac{1}{\sqrt{4 \pi}} \lim _{s \rightarrow 1} \sum_{\mathbf{n} \in \mathbb{Z}^{3}} \frac{1}{\left(\mathbf{n}^{2}-q^{2}\right)^{s}} .
$$

The same result can be easily obtained within the EFT approach, which has the advantage that it can be easily generalized to treat more complicated systems. Below we closely follow the line of the original paper [33]. Since the characteristic momenta on the lattice $p \sim L^{-1}$, the condition $M_{\pi} L \gg 1$ implies $p \ll M_{\pi}$. Systems with such small momenta can be described by the so-called non-relativistic EFT, which contains only particles (antiparticles are consistently included in the couplings of the Lagrangian). In other words, the particle number is a conserved quantity in the non-relativistic EFT, since the particle number nonconserving processes contain the large energy scale $M_{\pi}$.

In the theory which conserves the particle number, the structure of the Feynman diagrams is remarkably simple. Namely, in the infinite volume, the two-particle scattering amplitude $T$ is given by the sum of the bubble diagrams shown in Fig. 1 and, therefore, obeys the LippmannSchwinger equation

$$
T=V+V G_{0} T \text {. }
$$

Here, $V$ denotes the sum of all two-particle irreducible diagrams (the potential) and $G_{0}$ stands for the free twoparticle Green function.

Unlike Ref. [33], we retain the relativistic dispersion law $w(\mathbf{p})=\sqrt{m^{2}+\mathbf{p}^{2}}$ for all particles, even if one is working in the non-relativistic theory. In Refs. [23, 34] one can find a very detailed discussion of the covariant nonrelativistic approach. The one-particle propagator is given by

$$
D(p)=\frac{1}{2 w(\mathbf{p})} \frac{1}{w(\mathbf{p})-p_{0}-i 0} .
$$

As seen, this propagator contains only the particle pole. The advantage of this approach is that the kinematic relations hold automatically and should not be adjusted order by order. For example, the thresholds are always at the right place from the beginning. The price for this is a more complicated structure of the corresponding Feynman integrals. In particular, as shown in Refs. [23, 34], if one works out these integrals just by using dimensional regularization, the result does not obey the counting rules. In order to observe these rules, one has to apply the socalled threshold expansion [35], which boils down to the following prescription: 


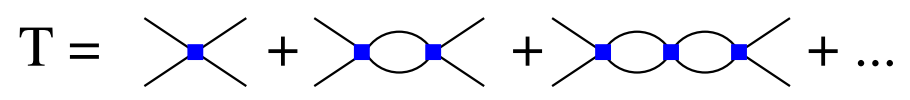

Figure 1. The sum of the bubble diagrams in the two-particle $T$-matrix in the non-relativistic EFT. The small filled square denotes the sum of all two-particle irreducible diagrams (the potential).

- Expand all integrands in the Feynman integrals in threemomenta;

- Perform the integration in the dimensional regularization order by order and sum up the result again.

As shown in Refs. [23, 34], after applying the threshold expansion, the Lippmann-Schwinger equation (3) turns into an algebraic equation. Indeed, the two-particle loop in the $\mathrm{CM}$ frame after the threshold expansion gives

$$
\begin{aligned}
& \int \frac{d^{D} k}{(2 \pi)^{D} i} \frac{1}{2 w_{1}(\mathbf{k}) 2 w_{2}(\mathbf{k})} \frac{1}{w_{1}(\mathbf{k})-k_{0}-i 0} \\
\times \quad & \frac{1}{w_{2}(\mathbf{k})-E+k_{0}-i 0}=\frac{i p}{8 \pi E} .
\end{aligned}
$$

Here, $E$ denotes the total energy of two particles in the CM frame $E=w_{1}(\mathbf{p})+w_{2}(\mathbf{p})$. Choosing a proper normalization and performing the partial-wave expansion, Eq. (3) in the $\mathrm{S}$-wave can be rewritten as

$$
T(p, p)=V(p, p)+i p V(p, p) T(p, p) .
$$

Consequently, in this scheme, $p V(p, p)=\tan \delta_{0}(p)$ denotes the on-shell $K$-matrix. It should be pointed out that the threshold expansion automatically "projects" all quantities, entering the Lippmann-Schwinger equation, onto the mass shell, since the off-shell effects lead to an regular integrand and vanish in this scheme. This constitutes a major advantage with respect to the relativistic approach, where, in some cases, checking the explicit cancellation of the off-shell effects is a rather non-trivial matter.

Next, we place the system in a three-dimensional box with a side length $L$ and calculate the finite-volume analog of the scattering $T$-matrix. Note that the momenta in a box are quantized:

$$
\mathbf{p}=\frac{2 \pi}{L} \mathbf{n}, \text { where } \mathbf{n} \in \mathbb{Z}^{3}
$$

Consequently, in all diagrams, the loop integrals will be replaced by discrete sums. On the other hand, the quantity $V$, which encodes the short-range physics related to the couplings of the effective Lagrangian, is not affected by the finite-volume artifacts up to exponentially suppressed terms $\propto \exp \left(-M_{\pi} L\right)$. The two-particle loop in a finite volume is given by

$$
\begin{gathered}
\frac{1}{L^{3}} \sum_{\mathbf{k}} \frac{1}{2 w_{1}(\mathbf{k}) 2 w_{2}(\mathbf{k})} \frac{1}{w_{1}(\mathbf{k})-k_{0}-i 0} \\
\times \frac{1}{w_{2}(\mathbf{k})-E+k_{0}-i 0}=\frac{1}{4 \pi^{3 / 2} E L} Z_{00}\left(1 ; q^{2}\right),
\end{gathered}
$$

and, if the partial-wave mixing is neglected, the finitevolume counterpart of Eq. (3) reads

$$
T_{L}(p, p)=V(p, p)+\frac{2}{\sqrt{\pi} L} Z_{00}\left(1 ; q^{2}\right) V(p, p) T_{L}(p, p) .
$$

In a finite volume, the quantity $T_{L}(p, p)$ possesses an infinite tower of poles, corresponding to the discrete eigenvalues of the full Hamiltonian. It is straightforward to ensure that the position of these poles is given exactly by Eq. (1). Thus, the Lüscher equation is reproduced in the EFT approach.

The above derivation clearly demonstrates the path, which is followed by the EFT approach in more complicated cases. The basis is given by the effective Lagrangian which is the same in a finite and in the infinite volume, up to exponentially suppressed corrections. This stems from the fact that the Lagrangian encodes the short-distance physics which is not affected by the infrared effects due to the presence of the box. Only the Feynman rules are modified, namely, the integrals are replaced by sums everywhere. The fact that the Lagrangian is the same enables one to relate the results of calculation in a finite and in the infinite volume and thus to establish the bridge between the observables we are looking for.

In the next sections, we shall consider three examples of such calculations. Namely, the resonance matrix elements and the three-particle states will be treated in detail, as well as the extraction of the resonance pole positions with the use of the UChPT.

\section{Resonance matrix elements}

As already mentioned in the Introduction, stable particles correspond to isolated energy levels of the Hamiltonian in a finite volume. Consequently, matrix elements of the weak and electromagnetic currents, evaluated between these eigenvalues, yield the pertinent form factors in the infinite-volume limit. The situation with resonances, which correspond to complex poles in the $S$-matrix, is different. It can be shown that the pertinent matrix elements, measured in a finite volume, do not have a well-defined infinite-volume limit. Hence, the following conceptual issues should be addressed:

1. Strictly speaking, one does not directly observe a resonance in the experiment, only the final products of its decay are detected. Is it possible to avoid the notion of a resonance whatsoever and to directly relate the decay amplitude to the results of the lattice measurements?

2. How does one define the resonance matrix element in the infinite volume and how does one measure it?

3. How does one extract this matrix element from the lattice data?

4. Do the procedures described in 1. and 3. agree in the limit of an infinitely narrow resonance? 


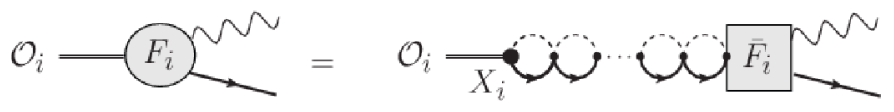

Figure 2. Matrix element in the EFT, exemplified on the decay $\Delta \rightarrow N \gamma^{*}$. The quantity $\bar{F}_{i}$ denotes the irreducible vertex. This quantity yields the full matrix element $F_{i}$ after dressing with any number of $\pi N$ bubbles that describe interactions in the initial state. The solid line with the operator $O_{i}$ denotes the resonance, here the delta, and solid, dashed, wiggly lines refer to nucleons, pions, and photons, respectively.

The answer to the question 1 . is provided by the socalled Lellouch-Lüscher formula [36], which relates the matrix element of a current, measured in a finite volume, to the matrix element of the same current in the infinite volume, evaluated between the scattering states that, in our case, emerge in the decay of a resonance (see also Ref. [37], where the moving frames were considered). In the original paper [36], the (non-resonant) weak decay process $K \rightarrow 2 \pi$ was considered. In Ref. [38], the approach was extended to the extraction of the timelike pion electromagnetic form factor, where the $\rho$ plays a prominent role. All these results can be most easily obtained within the EFT approach which, in addition, can be straightforwardly generalized to treat more complicated systems [11-14, 39-44].

The answer to question 2. is in fact well known since decades. Consider an arbitrary (local or non-local) scalar operator $O(x)$, which has the internal quantum numbers of a given resonance. The statement that a resonance is present is equivalent to the claim that the two-point function

$$
D\left(p^{2}\right)=i \int d^{4} x e^{i p x}\left\langle 0\left|T O(x) O^{\dagger}(0)\right| 0\right\rangle
$$

has a pole in the complex variable $z=p^{2}$ on the lower half of the second Riemann sheet at $z=p_{R}^{2}$ :

$$
\lim _{p^{2} \rightarrow p_{R}^{2}} D\left(p^{2}\right)=\frac{B_{R}}{p_{R}^{2}-p^{2}}+\cdots .
$$

The real and imaginary parts of $p_{R}^{2}$ are related to the resonance mass $m_{R}$ and the width $\Gamma_{R}$, according to $\operatorname{Re} p_{R}^{2}=$ $m_{R}^{2}-\Gamma_{R}^{2} / 4, \operatorname{Im} p_{R}^{2}=-m_{R} \Gamma_{R}$.

In order to define resonance matrix elements of, e.g., the electromagnetic current $J_{\mu}$, we consider the following three-point function:

$$
\begin{aligned}
& V_{\mu}(p, q) \\
= & -\int d^{4} x d^{4} y e^{i p x-i q y}\left\langle 0\left|T O(x) J_{\mu}(0) O^{\dagger}(y)\right| 0\right\rangle .
\end{aligned}
$$

The form factor of a resonance is then defined as

$$
\begin{aligned}
& i(p+q)_{\mu} F\left((p-q)^{2}\right) \doteq\left\langle p\left|J_{\mu}(0)\right| q\right\rangle \\
= & \lim _{p^{2}, q^{2} \rightarrow p_{R}^{2}} B_{R}^{-1 / 2}\left(p_{R}^{2}-p^{2}\right) V_{\mu}(p, q)\left(p_{R}^{2}-q^{2}\right) B_{R}^{-1 / 2},
\end{aligned}
$$

where $B_{R}$ is the residue at the resonance pole, see Eq. (11). Note that the matrix element displayed in Eq. (13) should be understood as a mere notation: in the spectrum, there exists no isolated resonance state with a definite momentum. Moreover, as it is clear from Eq. (13), this definition of the resonance matrix elements necessarily implies an analytic continuation into the complex plane. We would like to stress that we are not aware of any consistent fieldtheoretical prescription, where the analytic continuation would not be employed. The generalization to resonances with arbitrary spin is straightforward.

The key question to be addressed here is 3 . As demonstrated in Refs. [11-14], this can be done by using the non-relativistic EFT framework and evaluating the matrix elements both in a finite and in the infinite volume. An example of this procedure, corresponding to the decay process $\Delta \rightarrow N \gamma^{*}$, is given in Fig. 2. It is seen that the matrix element $F_{i}$ we are looking for is given as a product of the two-particle irreducible vertex $\bar{F}_{i}$ and any number of two-particle bubbles. The difference between the two cases is concentrated exactly in these bubbles, because the two-particle irreducible blocks are exactly the same in a finite and in the infinite volume (the finite-volume effects are exponentially suppressed). Moreover, the analytic continuation in the irreducible blocks can be done by using an analog of the effective-range expansion, because these blocks can be expressed in terms of low-energy polynomials. Finally, one may formulate a unique prescription, which relates the matrix element, measured on the lattice, to the resonance matrix element evaluated at the pole. We do not give the details of this prescription here. The interested reader can find these details in Refs. [11-14]. Moreover, it can be directly shown that, for infinitely narrow resonances, the matrix elements measured on the lattice coincide with the ones extracted by using this procedure.

There is, however, one complication which should also be mentioned. If a resonance appears both in the initial and the final states (an example: the electromagnetic form factor of the $\Delta$, see Ref. [12]), the triangle diagram becomes the source of a problem that hinders an analytic continuation to the resonance pole (the so-called finite fixed points emerge [12]). In this case, one may use lattice measurements of the matrix element between two nearby eigenstates in order to cancel the contribution of the triangle diagram.

Finally, note that the analytic continuation is performed in the CM frame, for a fixed momentum transfer three-vector. After analytic continuation, the fourth component of the momentum transfer becomes complex, as well as the virtuality $q^{2}$. Albeit at present neither the experimental nor the lattice collaborations provide the values of the form factors at complex virtuality, we believe that this is the only consistent way of presenting the results. The form factors defined at real virtuality, in general, are off the pole (the elastic form factors in the Breit frame 

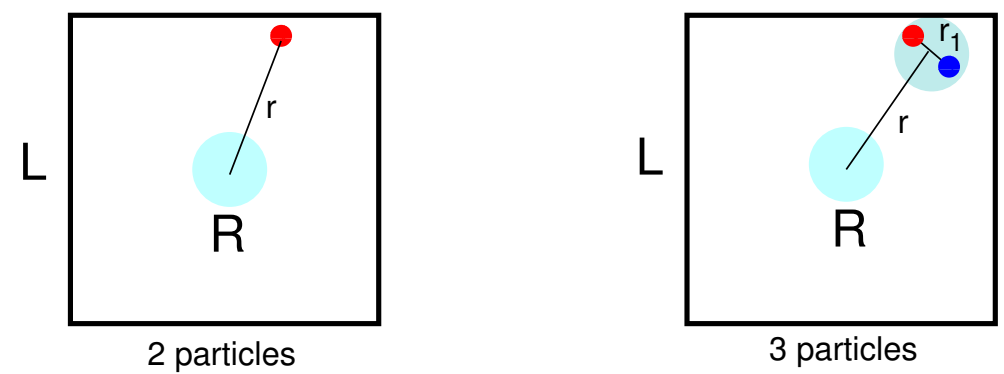

Figure 3. Two- and three-particle systems, placed in a finite box. In case of the two-particle system, if the box size $L$ is much larger than the interaction range $R$, the particle close to the box experiences the asymptotic wave function only. Matters are different for the three-particle system, even if $L \gg R$, two particles close to the wall can experience strong interactions.

are an exception). Consequently, the background does not vanish and the result will be process-dependent.

\section{Three particles in a finite volume}

The rapid advance of calculations of the properties of various nuclei on the lattice invokes the necessity of the study of the many-body systems in a finite volume. An obvious candidate to start with is the three-body system, as studied e.g. in project B.6. Another justification is provided by the necessity of the proper treatment of the inelastic resonances, e.g., the Roper resonance, whose decay rate into the three-particle final state is sizable (quark mass expansions for the Roper are studied in projects $\mathbf{C . 3}$ and $\mathbf{C . 9}$ ).

The conceptual difference between the two-particle and three-particle problems in a finite volume is clearly demonstrated in Fig. 3. In the two-particle case, after removing the CM motion, we are left with the effective oneparticle problem. If the interaction range is much smaller than the size of the box, the effective particle in the vicinity of the walls is necessarily far away from the interaction region, i.e., the wave function nearby the walls can be replaced by the asymptotic wave function. This means that the boundary conditions, which determine the spectrum in a finite volume, contain the asymptotic wave function only - in other words, the spectrum in a finite volume is solely determined by the $S$-matrix elements in the infinite volume.

The situation is different in the case of the threeparticle system. Again, after removing the CM motion, we are left with two effective particles. However, in this case, these two particles may be close to the walls and to each other simultaneously, even if the interaction range is much smaller than the size of the box. For this reason, it is not $a$ priori clear that the three-body spectrum in a finite volume is solely determined by the pertinent $S$-matrix elements.

The study of the three-body systems in a finite volume is still in its infancy. Below, we list some of the major achievements in the last few years:

- In the pioneering work [15], it has been demonstrated that the finite-volume spectrum is indeed determined solely by the infinite-volume $S$-matrix despite the problem mentioned above.
- In Refs. [17-19], the spectrum of the three-particle system in a finite volume has been studied by solving the EFT equations numerically. The volume-dependence of the energy levels is obtained. For details of the formalism in the infinite volume, see the contribution from project B.6.

- In Refs. [45-48], the three-particle quantization condition (an analog of the Lüscher equation in the twoparticle systems) has been obtained and explored for various simple systems.

- In Ref. [20], an explicit expression for the energy shift of the three-body bound state in a finite volume in the unitary limit (infinitely large two-body scattering length and vanishing effective range) was obtained. The expression has the following form:

$$
\frac{\Delta E}{E}=\mathrm{const}|A|^{2}(\kappa L)^{-3 / 2} \exp \left(-\frac{2}{\sqrt{3}} \kappa L\right),
$$

where $A$ stands for the asymptotic normalization coefficient of the three-particle wave function, $E$ is the binding energy, $m$ is the particle mass and $\kappa=\sqrt{m E}$ denotes the three-body binding momentum.

There is still a host of interesting issues which should be addressed. In particular, it would be interesting to see, how the above explicit result can be obtained by using the general three-body quantization condition from Refs. [4548].

\section{Insights from UChPT in a finite volume}

The extraction of the scalar meson resonance parameters is particularly challenging, not only in Nature but also in a finite volume. Unitarized Chiral Perturbation Theory (UChPT) provides a successful approach that reproduces well the $\pi \pi / \pi \eta$ and $K \bar{K}$ data up to $1200 \mathrm{MeV}$. In this scheme, the resonances $f_{0}(600), f_{0}(980), a_{0}(980)$ are generated dynamically from the coupled channel interaction, see e.g. Refs. [49-51].

In Ref. [6] we have discussed the extraction of the mass and width of the scalar mesons $f_{0}(980)$ and $a_{0}(980)$ from the finite-volume spectrum in lattice QCD. In particular, it is argued that the nature of these states can be studied by invoking twisted boundary conditions, as well 
as investigating the quark mass dependence of the spectrum. It was further shown that a two-particle threshold can mimic the occurrence of a resonance through the appearance of an avoided level crossing, see Fig. 4.

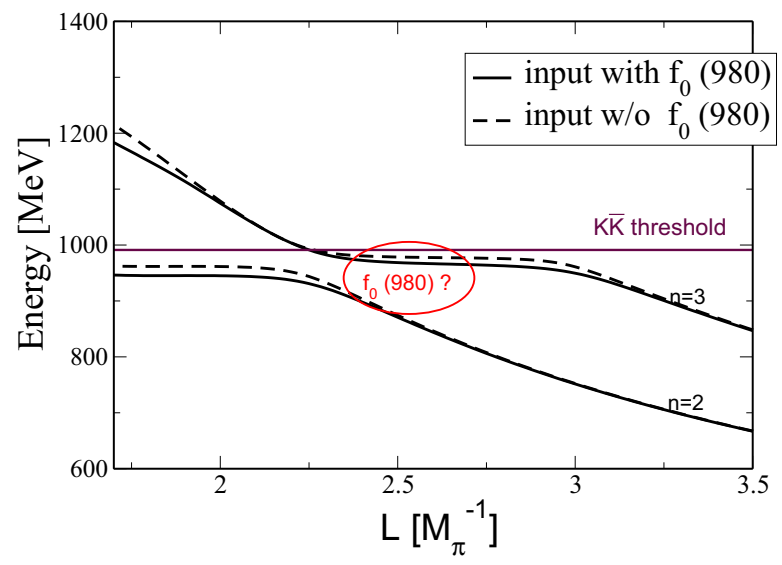

Figure 4. Energy levels calculated in finite volume UChPT for the $\pi \pi / K \bar{K}$ system in the vicinity of the $K \bar{K}$ threshold and the $f_{0}(980)$ resonance (solid lines). Even if one removes the scalar resonance, the avoided level crossing still appears (dashed lines).

In the paper [7], we have discussed the extraction of the parameters of the scalar mesons $f_{0}(600), f_{0}(980)$ and $a_{0}(980)$ resonances from lattice data. In order to facilitate this extraction, we have used UChPT in a finite volume in the fit. Fitting synthetic data, we have demonstrated that the approach works, and the pole position for the above resonances can indeed be extracted by analyzing the volume-dependent energy spectrum in the vicinity of the resonance energy, provided sufficiently many volumes are simulated. The different sources of errors were analyzed in detail. The key point is that the use of the phenomenological input from UChPT stabilizes the fit. It is, thus, very challenging to apply these results to present and forthcoming lattice data in order to extract valuable information concerning hadronic resonances. From the theoretical point of view one should also keep in mind that in field theory there are parts of the kernel of the Bethe-Salpeter equation which are volume dependent, although exponentially suppressed. Quantitative studies of these terms and the extent of the large $L$ suppression would be a very good complement to the work we have carried out in that work.

Dynamical coupled-channel approaches are a widely used tool in hadronic physics that allow to analyze different reactions and partial waves in a consistent way. In such approaches the basic interactions are derived within an effective Lagrangian framework and the resulting pseudopotentials are then unitarized in a coupled-channel scattering equation. In Ref. [8], we have proposed a scheme that allows for a solution of the arising integral equation in discretized momentum space for periodic as well as twisted boundary conditions. This permits to study finite size effects as they appear in lattice QCD simulations. The new formalism, at this stage with a restriction to S-waves, was applied to coupled-channel models for the light scalar mesons, and also for the $\Lambda(1405)$ baryon. Lattice spectra were predicted, see Fig. 5 for the coupled $\bar{K} N-\pi \Sigma$ systems that features the $\Lambda(1405)$ resonance.

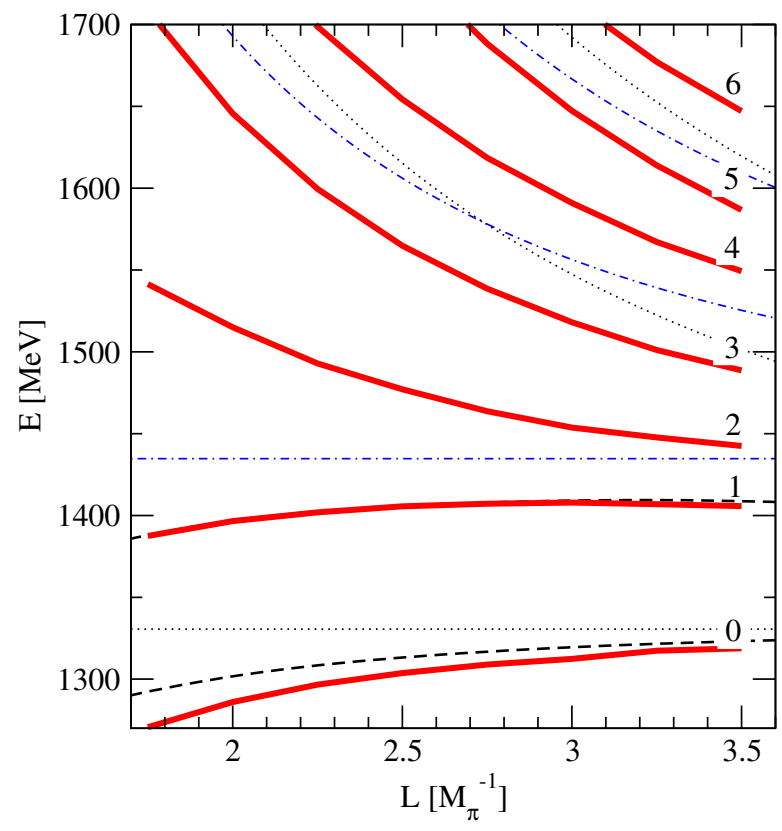

Figure 5. Spectrum $E(L)$ of the $I=0, S=-1, J^{P}=\frac{1}{2}^{-}$mesonbaryon sector $[\Lambda(1405)]$ for periodic boundary conditions. Solid lines: results for the $\bar{K} N$ Jülich model of hadron exchange [52]. Dashed lines: For comparison, the first two levels as obtained from the chiral unitary approach of Ref. [53]. Dotted (dashdotted) lines: free $\pi \Sigma(\bar{K} N)$ levels.

In the study [9] we have provided a formulation for the scattering of two particles confined in a finite box with total nonzero momentum, adapted to the UChPT framework. This extended previously known techniques for zero momentum, discretizing the energy levels by imposing the boundary conditions in the moving frame. Given a hadronic interaction, we have predicted levels for the first five boosts $\vec{P}=(2 \pi / L)(0,0,0)$ to $(0,0,2)$ and attributed these to the subgroups of cubic symmetry. Employing UChPT including NLO terms, we have derived the levels for the mixed-partial wave system with $I=1 / 2$, $S=-1$ and $L=0,1\left[\kappa(800)\right.$ and $K^{*}(892)$, respectively] as well as for the scalar sector with $I=0, S=0$ and $L=0,2$ where the $\sigma(600)$ resides. We have demonstrated for the $\kappa(800) / K^{*}(892)$ system that partial wave mixing is a very large effect for realistic box sizes and needs to be taken into account. To disentangle the S-wave from the P- or D-wave, we have derived a set of equations in the one-channel formalism that are shown to be very precise as long as one stays below the inelastic thresholds. Furthermore, we have worked out a scheme in which the hadronic interaction is expanded in energy to allow for the extraction of the infinite volume limit, simultaneously fitting levels for different boosts and at different energies. The model-independent information from the lowest order in the chiral expansion is kept explicitly in this expansion, greatly stabilizing the fit to lattice data. Such statistical analyses can be used for actual lattice data, or, as done here, serve to determine promising lattice setups and the 
accuracy of lattice data to allow for reliable resonance extraction. We have tested this new method for the example of the $\sigma(600)$. We conclude that using information from moving frames is indeed rewarding since, with only a few different box sizes, phase shifts and resonance parameters of excited mesons can be determined.

Simulating the $\kappa(800)$ on the lattice is a challenging task that starts to become feasible due to the rapid progress in recent-years lattice QCD calculations. As the resonance is broad, special attention to finite-volume effects has to be paid, because no sharp resonance signal as from avoided level crossing can be expected. In the paper [10], we have investigated the finite volume effects in the framework of unitarized chiral perturbation theory using nextto-leading order terms. After a fit to meson-meson partial wave data, lattice levels for $\pi K$ scattering are predicted. In addition, levels are shown for the quantum numbers in which the $\sigma(600), f_{0}(980), a_{0}(980), \phi(1020), K^{*}(892)$, and $\rho(770)$ appear, as well as the repulsive channels. Methods to extract the $\kappa(800)$ signal from the lattice spectrum are presented. Using pseudo-data, we estimate the precision that lattice data should have to allow for a clear-cut extraction of this resonance. To put the results into context, in particular the required high precision on the lattice data, the $\sigma(600)$, the P-wave resonances $K^{*}(892)$ and $\rho(770)$, and the repulsive $\pi K, \pi \pi$ phases were analyzed as well.

\section{Conclusions, outlook}

The SFB/TR 16 was finished at the end of June 2016. However, the investigations, which were launched within this project, are continued within other projects, part of which are funded by the DFG (e.g. within the CRC 110 "Symmetries and the Emergence of Structure in QCD"). In this respect, we would like to especially mention the study of the electromagnetic and weak matrix elements in a finite volume, as well as the extensive investigations of the three-particle problem.

The work reported here would not have been possible without the support from the Deutsche Forschungsgemeinschaft within the SFB/TR16. We would like to thank all our collaborators, who have contributed to this work: A. Agadjanov, D. Agadjanov, V. Baru, V. Bernard, M. Bissegger, S. Bour, G. Colangelo, M. Döring, E. Epelbaum, A. Fuhrer, J. Gasser, M. Göckeler, F.-K. Guo, C.O. Güllstrom, H.-W. Hammer, J. Haidenbauer, Ch. Hanhart, D. Hoja, R. Horsley, A. Khelashvili, S. König, H. Krebs, S. Kreuzer, B. Kubis, A. Kupsc, M. Lage, D. Lee, F. LlanesEstrada, V.E. Lyubovitskij, E. Oset, K. Polejaeva, P.E.L. Rakow, G. Rios, G. Schierholz and J.M. Zanotti.

\section{References}

[1] M. Lüscher, Nucl. Phys. B 354, 531 (1991).

[2] V. Bernard, D. Hoja, U.-G. Meißner and A. Rusetsky, JHEP 0906,061 (2009).

[3] V. Bernard, M. Lage, U.-G. Meißner and A. Rusetsky, JHEP 0808, 024 (2008).

[4] M. Göckeler, R. Horsley, M. Lage, U.-G. Meißner, P. E. L. Rakow, A. Rusetsky, G. Schierholz and J. M. Zanotti, Phys. Rev. D 86, 094513 (2012).
[5] M. Lage, U.-G. Meißner and A. Rusetsky, Phys. Lett. B 681, 439 (2009).

[6] V. Bernard, M. Lage, U.-G. Meißner and A. Rusetsky, JHEP 1101, 019 (2011).

[7] M. Döring, U.-G. Meißner, E. Oset and A. Rusetsky, Eur. Phys. J. A 47, 139 (2011).

[8] M. Döring, J. Haidenbauer, U.-G. Meißner and A. Rusetsky, Eur. Phys. J. A 47, 163 (2011).

[9] M. Döring, U.-G. Meißner, E. Oset and A. Rusetsky, Eur. Phys. J. A 48, 114 (2012).

[10] M. Döring and U.-G. Meißner, JHEP 1201, 009 (2012).

[11] D. Hoja, U.-G. Meißner and A. Rusetsky, JHEP 1004, 050 (2010).

[12] V. Bernard, D. Hoja, U.-G. Meißner and A. Rusetsky, JHEP 1209, 023 (2012).

[13] A. Agadjanov, V. Bernard, U.-G. Meißner and A. Rusetsky, Nucl. Phys. B 886, 1199 (2014).

[14] A. Agadjanov, V. Bernard, U.-G. Meißner and A. Rusetsky, Nucl. Phys. B 910, 387 (2016).

[15] K. Polejaeva and A. Rusetsky, Eur. Phys. J. A 48, 67 (2012).

[16] S. Bour, S. Koenig, D. Lee, H.-W. Hammer and U.G. Meißner, Phys. Rev. D 84, 091503 (2011).

[17] S. Kreuzer and H.-W. Hammer, Phys. Lett. B 673, 260 (2009).

[18] S. Kreuzer and H.-W. Hammer, Eur. Phys. J. A 43, 229 (2010).

[19] S. Kreuzer and H.-W. Hammer, Phys. Lett. B 694, 424 (2011).

[20] U.-G. Meißner, G. Rios and A. Rusetsky, Phys. Rev. Lett. 114, 091602 (2015).

[21] M. Bissegger, A. Fuhrer, J. Gasser, B. Kubis and A. Rusetsky, Nucl. Phys. B 806, 178 (2009).

[22] C.-O. Güllstrom, A. Kupsc and A. Rusetsky, Phys. Rev. C 79, 028201 (2009).

[23] J. Gasser, B. Kubis and A. Rusetsky, Nucl. Phys. B 850, 96 (2011).

[24] G. Colangelo, J. Gasser and A. Rusetsky, Eur. Phys. J. C 59, 777 (2009).

[25] J. Gasser, V. E. Lyubovitskij and A. Rusetsky, Ann. Rev. Nucl. Part. Sci. 59, 169 (2009).

[26] V. Baru, E. Epelbaum and A. Rusetsky, Eur. Phys. J. A 42, 111 (2009).

[27] F. K. Guo, C. Hanhart, F. J. Llanes-Estrada and U.G. Meißner, Phys. Lett. B 678, 90 (2009).

[28] F.-K. Guo, C. Hanhart, F. J. Llanes-Estrada and U.G. Meißner, Phys. Lett. B 703, 510 (2011).

[29] H. Krebs, E. Epelbaum and U.-G. Meißner, Phys. Rev. C 80, 028201 (2009).

[30] H. Krebs, E. Epelbaum and U.-G. Meißner, Phys. Lett. B 683, 222 (2010).

[31] A. Agadjanov, D. Agadjanov, A. Khelashvili and A. Rusetsky, Eur. Phys. J. A 49, 120 (2013).

[32] U.-G. Meißner, K. Polejaeva and A. Rusetsky, Nucl. Phys. B 846, 1 (2011). 
[33] S. R. Beane, P. F. Bedaque, A. Parreno and M. J. Savage, Nucl. Phys. A 747, 55 (2005).

[34] G. Colangelo, J. Gasser, B. Kubis and A. Rusetsky, Phys. Lett. B 638, 187 (2006).

[35] M. Beneke and V. A. Smirnov, Nucl. Phys. B 522, 321 (1998).

[36] L. Lellouch and M. Lüscher, Commun. Math. Phys. 219, 31 (2001).

[37] C. h. Kim, C. T. Sachrajda, S. R. Sharpe, Nucl. Phys. B 727, 218 (2005).

[38] H. B. Meyer, Phys. Rev. Lett. 107, 072002 (2011).

[39] M. T. Hansen and S. R. Sharpe, Phys. Rev. D 86, 016007 (2012).

[40] R. A. Briceno, M. T. Hansen and A. Walker-Loud, Phys. Rev. D 91, 034501 (2015).

[41] R. A. Briceno and M. T. Hansen, Phys. Rev. D 92 , 074509 (2015).

[42] R. A. Briceno, J. J. Dudek, R. G. Edwards, C. J. Shultz, C. E. Thomas and D. J. Wilson, Phys. Rev. Lett. 115, 242001 (2015).
[43] R. A. Briceno and M. T. Hansen, arXiv:1509.08507 [hep-lat].

[44] R. A. Briceno, J. J. Dudek, R. G. Edwards, C. J. Shultz, C. E. Thomas and D. J. Wilson, Phys. Rev. D 93, 114508 (2016).

[45] M. T. Hansen and S. R. Sharpe, Phys. Rev. D 90, 116003 (2014).

[46] M. T. Hansen and S. R. Sharpe, Phys. Rev. D 92, 114509 (2015).

[47] M. T. Hansen and S. R. Sharpe, Phys. Rev. D 93, 014506 (2016).

[48] M. T. Hansen and S. R. Sharpe, Phys. Rev. D 93, 096006 (2016).

[49] J. A. Oller, E. Oset, Nucl. Phys. A620 (1997) 438 [Erratum-ibid. A 652 (1999) 407].

[50] J. R. Pelaez, Phys. Rev. Lett. 92 (2004) 102001.

[51] N. Kaiser, Eur. Phys. J. A3 (1998) 307.

[52] J. Haidenbauer, G. Krein, U.-G. Meißner and L. Tolos, Eur. Phys. J. A 47 (2011) 18.

[53] E. Oset and A. Ramos, Nucl. Phys. A 635 (1998) 99. 